\title{
Characteristics of Cyclin B and its potential role in regulating oogenesis in the red claw crayfish (Cherax quadricarinatus)
}

\author{
L.M. Wang ${ }^{1,2}$, W.W. Lv ${ }^{2}$, D. Zuo ${ }^{2}$, Z.J. Dong ${ }^{1}$ and Y.L. Zhao ${ }^{2}$ \\ ${ }^{1}$ Key Laboratory of Freshwater Fisheries and Germplasm Resources Utilization, \\ Ministry of Agriculture, Freshwater Fisheries Research Center of Chinese \\ Academy of Fishery Sciences, Wuxi, China \\ ${ }^{2}$ Life Science College, East China Normal University, Shanghai, China \\ Corresponding authors: Y.L. Zhao / Z.J. Dong \\ E-mail: ylzhao@bio.ecnu.edu.cn / dongzj@ffrc.cn
}

Genet. Mol. Res. 14 (3): 10786-10798 (2015)

Received February 10, 2015

Accepted May 15, 2015

Published September 9, 2015

DOI http://dx.doi.org/10.4238/2015.September.9.17

\begin{abstract}
Cyclin B is a regulatory subunit of maturation-promoting factor (MPF), which has a key role in the induction of meiotic maturation of oocytes. MPF has been studied in a wide variety of animal species; however, its expression in crustaceans is poorly characterized. In this study, the complete cDNA sequence of Cyclin B was cloned from the red claw crayfish, Cherax quadricarinatus, and its spatiotemporal expression profiles were analyzed. Cyclin B cDNA (1779 bp) encoded a 401 amino acid protein with a calculated molecular weight of $45.1 \mathrm{kDa}$. Quantitative real-time PCR demonstrated that Cyclin B mRNA was expressed mainly in the ovarian tissue and that the expression decreased as the ovaries developed. Immunofluorescence analysis revealed that the Cyclin B protein relocated from the cytoplasm to the nucleus during oogenesis. These findings suggest that Cyclin B plays an important role in gametogenesis and gonad development in C. quadricarinatus.
\end{abstract}

Key words: Cyclin B; cDNA cloning; Cellular localization; Spatiotemporal expression profile; Oogenesis; Cherax quadricarinatus 


\section{INTRODUCTION}

The red claw crayfish, Cherax quadricarinatus (von Martens, 1868), is a crustacean species commercially farmed in several countries worldwide because of its potential size, tolerance to a broad range of temperatures and water quality conditions, and large market potential. Over the past decades, considerable progress has been made in the study of its reproductive biology (Sagi et al., 1996; Abdu et al., 2000, 2002; Rodríguez-González et al., 2006; Li et al., 2007, 2010, 2011; Vazquez et al., 2008; Wang et al., 2013a), but the molecular mechanisms of oocyte maturation are rarely studied (Wang et al., 2013b). Hence, a better understanding of these molecular mechanisms is important for the artificial control of breeding and could promote large-scale industrial culture in China.

Cyclin B is a prevalent regulatory protein in cells. Progression through the cell cycle is regulated by a complex of cyclin-dependent kinase 2 (Cdc2) and cyclin B (also called maturation or M-phase promoting factor, MPF), which allows cells to proceed through the G2-M checkpoint (Minshull et al., 1990; Lees and Harlow, 1993; Li et al., 2004). Although the role of MPF in oocyte maturation has been studied in a wide variety of animal species, little information is available for crustaceans. Thus far, the molecular characteristics of crustacean Cdc2 kinase have only been reported for Eriocheir sinensis (Qiu and Liu, 2009), Macrobrachium nipponense (Wu, 2009), and C. quadricarinatus (Wang et al., 2013b). Cyclin B transcripts have been identified and characterized in Marsupenaeus japonicus (Qiu and Yamano, 2005), E. sinensis (Fang and Qiu, 2009), Penaeus monodon (Qiu et al., 2007; Visudtiphole et al., 2009), M. nipponense (Wu, 2009), and Macrobrachium rosenbergii (Fang, 2008).

After characterizing the, spatiotemporal expression profiles and cellular localization of C. quadricarinatus Cdc2 kinase (Wang et al., 2013b), we cloned, for the first time, the complete cDNA sequence of Cyclin B from C. quadricarinatus, and we evaluated its temporal and spatial expression profiles during ovarian and embryonic development. In order to understand the role of Cyclin B in oocyte maturation in crustaceans, we then examined the localization of the Cyclin B protein during oogenesis by immunofluorescence.

\section{MATERIAL AND METHODS}

\section{Sample preparation}

C. quadricarinatus crayfish were supplied by the CaoJing Aquaculture Demonstration Garden Hatchery (Shanghai, China) and purchased from a local shrimp farm. A total of 100 female and 10 male crayfish (initial weight $=20.64 \pm 1.53 \mathrm{~g}$ ) were reared indoors in 10 and $1 \times 300-\mathrm{L}$ plastic containers separately under controlled conditions $\left[27 \pm 1^{\circ} \mathrm{C} ; \mathrm{pH}=6.5-7.5\right.$, dissolved oxygen $(\mathrm{DO})>6 \mathrm{mg} / \mathrm{L}$ and 10:14 dark:light photoperiod] for 90 days. Two tiles and PVC tubes (5-cm diameter, 20-cm length) were distributed as shelters along the bottom of the containers. The crayfish were fed commercial pellets (Hongma Feed Company, Shanghai, China) containing $35 \%$ crude protein and $8 \%$ lipid, which is regarded as an optimum diet for gonadal development (Rodríguez-González et al., 2009a,b). After 15 days of feeding adaptation, 10 females (intermolt) were randomly collected at regular intervals (15 days) and weighed. Wounded or incomplete specimens were discarded. The crayfish were then placed in an ice bath for 1-2 min until they were lightly anesthetized, and were sacrificed to obtain various tissues, including ovaries (OV), testes (TT), antennal gland (AG), muscle (MU), he- 
patopancreas (HP), hemocytes (HC), intestine (IT), and brain (B). The tissue samples were snap-frozen in liquid nitrogen and conserved at $-80^{\circ} \mathrm{C}$ for subsequent nucleic acid analysis.

In addition, ovaries were dissected and weighed to calculate the gonadosomatic index (GSI) values (wet weight of the gonads/total body weight x 100) and fixed in $0.01 \mathrm{M}$ phosphate-buffered saline (PBS) containing $4 \%$ paraformaldehyde at $4^{\circ} \mathrm{C}$ for $12 \mathrm{~h}$. After washing with PBS three times, the ovaries were dehydrated in 10, 20, and 30\% saccharose-PBS solutions, respectively, at $4^{\circ} \mathrm{C}$, and then embedded in Tissue-Tek Optimal Cutting Temperature compound (Sakure, USA). Standard frozen sections 8 - $\mu \mathrm{m}$-thick were produced using a microtome (Leica, Germany). The sections were used for immunofluorescence (IF) analyses and conventional histological observation to determine developmental stages.

Ovarian development was classified into four stages according to ovarian color changes and GSI, as previously described (Wang et al., 2013b). The ovarian stage of each crayfish was further confirmed by conventional histology (hematoxylin/eosin staining). Two male crayfish were placed into a container in which the female crayfish had a mature ovary. After a spermatophore was observed on the female reproductive hole, the male was removed. The fertilized eggs and embryos were classified into five stages as previously described (Wang et al., 2013b). Specimens were placed in liquid nitrogen and stored at $-80^{\circ} \mathrm{C}$ for subsequent total RNA extraction and quantitative real-time PCR (qRT-PCR) analysis. Three biological samples $(\mathrm{N}=3)$ were analyzed.

\section{Nucleic acid preparation and first-strand cDNA synthesis}

Total RNA was extracted from various tissues using Tissue RNA Rapid Extraction Kit (Yuanpinghao Biotech Co., Ltd., Tianjin, China) according to the manufacturer protocol. The quantity and quality of RNA was examined by UV-spectrophotometry $\left(\mathrm{OD}_{260} / \mathrm{OD}_{280}\right)$ and agarose gel electrophoresis, respectively. Total RNA (500 ng) from various tissues was reverse transcribed into first-strand cDNA using a PrimeScript RT Master Mix Perfect Real Time Kit (TaKaRa, Japan) according to the manufacturer instructions.

\section{Degenerate RT-PCR (reverse transcription polymerase chain reaction)}

The degenerate primers Cyclin B-F and Cyclin B-R (Table 1) were designed based on a highly conserved domain from known Cyclin B gene sequences, and were used to amplify the target fragment of the Cyclin B gene. First-strand cDNA was subjected to PCR amplification using Premix Ex Taq ${ }^{\mathrm{TM}}$ Hot Start Version (TaKaRa, Japan), and degenerate primers (20 $\mu \mathrm{M}$ ) were used at final concentrations in a $25-\mu \mathrm{L}$ reaction for $94^{\circ} \mathrm{C}$ for $2 \mathrm{~min}$, followed by 35 cycles of $98^{\circ} \mathrm{C}$ for $10 \mathrm{~s}, 40^{\circ} \mathrm{C}$ for $30 \mathrm{~s}, 72^{\circ} \mathrm{C}$ for $1 \mathrm{~min}$, and a final elongation step at $72^{\circ} \mathrm{C}$ for 6 min. PCR products were separated on 1.5\% agarose gel and purified with an E.Z.N.A ${ }^{\circledR}$ Gel Extraction Kit (Omiga BioTek, USA). The purified PCR products were cloned into a pMD19-T vector (TaKaRa) overnight. The recombinant plasmid was transformed into competent Escherichia coli cells and positive clones were sequenced at Shanghai Biosune Bio Co. Ltd., China. The retrieved sequences were verified and compared with those of other known Cyclin B sequences using the BLASTX program at the National Center for Biotechnology Information (http://www.ncbi.nlm.nih.gov/blast). 


\begin{tabular}{|c|c|c|}
\hline Primers & Sequences & Code \\
\hline \multicolumn{3}{|c|}{ Degenerate primers to target Cyclin B } \\
\hline Cyclin B Forward & 5'-ATWGCWAGYAAATAYGAAGARA-3' & DP-F \\
\hline Cyclin B Reverse & 5'-TCCATIARRTAYTTKGCWARIGTA-3' & DP-R \\
\hline \multicolumn{3}{|c|}{ Primers for 5'-RACE and 3'-RACE PCR } \\
\hline Cyclin B 5'-RACE primer & 5'-GGCTAGGGTGTGTTGGGCTGCATCA-3' & $5 '-R$ \\
\hline Cyclin B 3'-RACE primer & 5'-CACCAAAAATGAAATTCGCAAAATGGA-3' & $3 '-R$ \\
\hline \multicolumn{3}{|l|}{ Primers for qRT-PCR } \\
\hline Cyclin B Forward & 5'-GAAATTCGCAAAATGGAAGTAAA-3' & Q-F \\
\hline Cyclin B Reverse & 5'-ATTTGGCTAGGGTGTGTTGG-3' & Q-R \\
\hline 18S rRNA Forward & 5'-CATGCCCGTTCTTAGTTGGT-3' & $18 \mathrm{~S}-\mathrm{F}$ \\
\hline 18S rRNA Reverse & 5'-GTGCGGCCCAGAAATATAAA-3' & $18 \mathrm{~S}-\mathrm{R}$ \\
\hline
\end{tabular}

\section{Rapid amplification of cDNA ends (RACE)}

Full-length Cyclin B cDNA was obtained by 5'- and 3'-RACE using the SMARTerTMRACE cDNA Amplification Kit (Clontech, USA) according to the manufacturer protocol. The 5'- and 3'-RACE primers (Table 1) were designed based on the cDNA sequence obtained for Cyclin B. The 3'-RACE PCR program was performed for 25 cycles of $94^{\circ} \mathrm{C}$ for $30 \mathrm{~s}, 68^{\circ} \mathrm{C}$ for $30 \mathrm{~s}$, and $72^{\circ} \mathrm{C}$ for $3 \mathrm{~min}$. The $5^{\prime}$-RACE program was performed by touchdown PCR for five cycles of $94^{\circ} \mathrm{C}$ for $30 \mathrm{~s}, 72^{\circ} \mathrm{C}$ for $3 \mathrm{~min}$, followed by five cycles of $94^{\circ} \mathrm{C}$ for 30 s, $70^{\circ} \mathrm{C}$ for $30 \mathrm{~s}, 72^{\circ} \mathrm{C}$ for $3 \mathrm{~min}$, and a final step at 25 cycles of $94^{\circ} \mathrm{C}$ for $30 \mathrm{~s}, 68^{\circ} \mathrm{C}$ for $30 \mathrm{~s}$, and $72^{\circ} \mathrm{C}$ for $3 \mathrm{~min}$. The amplified cDNA fragments were cloned and sequenced as described for degenerate RT-PCR above.

\section{Multiple sequence alignment and phylogenetic analysis}

Deduced protein sequences of Cyclin B from various species were retrieved from GenBank and phylogenetically compared with those of C. quadricarinatus. Multiple alignments were carried out using ClustalX. A bootstrapped neighbor-joining phylogenetic tree was constructed using the MEGA software version4.0 (http://www.megasoftware.net/). The reliability of the branching was tested using bootstrap re-sampling with 1000 pseudo replicates.

\section{qRT-PCR analysis}

mRNA expression was analyzed by qRT-PCR during the tissue-dependent and different stages of ovarian and embryonic development, according to the following method. Genespecific primers (Table 1) were designed based on the cloned Cyclin B cDNA sequence to produce an amplicon of $133 \mathrm{bp}$. qRT-PCR was performed in a C1000 ${ }^{\mathrm{TM}}$ Thermal Cycler (BioRad CFX 96 ${ }^{\mathrm{TM}}$ Real-Time System) according to the manufacturer protocol. The final volume of each qRT-PCR reaction was $25 \mu \mathrm{L}$, which contained $12.5 \mu \mathrm{L}$ 2X SYBR Premix ExTaq (TaKaRa, Japan), $1.0 \mu \mathrm{L}$ diluted cDNA template (100 ng RNA), $9.5 \mu \mathrm{L}$ PCR-grade water, and $1.0 \mu \mathrm{L}$ of each $10 \mu \mathrm{M}$ primer. PCR conditions were as follows: $95^{\circ} \mathrm{C}$ for $30 \mathrm{~s}$, followed by 40 cycles of $95^{\circ} \mathrm{C}$ for $5 \mathrm{~s}$, and $59^{\circ} \mathrm{C}$ for $30 \mathrm{~s}$. Each sample was run in triplicate and the results were normalized to the selected control gene 18S rRNA of C. quadricarinatus (AF235966). The primers of $18 \mathrm{~S}$ rRNA (Table 1) were designed to produce an amplicon of $176 \mathrm{bp}$.

Cyclin B expression levels were calculated by the $2^{-\Delta \Delta \mathrm{Ct}}$ comparative CT method ( $\mathrm{Li}$ - 
vak and Schmittgen, 2001). Mean and standard deviations were calculated from triplicate experiments, and presented as $n$-fold differences in expression relative to $18 \mathrm{~S}$ rRNA. Data were analyzed using the CFX Manager ${ }^{\mathrm{TM}}$ software (version 1.0). Data are reported as mean values \pm standard error (SE). The homogeneity of variance was confirmed and comparison between means was performed with a one-way ANOVA. Duncan's procedure was used for multiple comparisons between groups. Differences were regarded as significant when $\mathrm{P}<0.05$. All statistical analyses were performed by STATISTICA 8.0 (Statsoft, USA).

\section{Immunofluorescence}

Immunofluorescence (IF) was carried out using Fast Tissue ImmunoFluorescence $^{\circledR}$ Staining Kit (The Protein Biotechnologies Company, Portland, USA) according to the manufacturer protocol. Briefly, after washing three times, frozen sections were incubated overnight at $4{ }^{\circ} \mathrm{C}$ with Cyclin $\mathrm{B}$ rabbit monoclonal antibody (Epitomics, USA) (1:100), and were then rinsed three times. A preliminary test confirmed that the Cyclin B rabbit monoclonal antibody was specific for Cyclin B in C. quadricarinatus. Subsequently, the tissue sections were incubated with a secondary antibody (goat anti-rabbit $\operatorname{IgG}$ ) conjugated with horseradish peroxidase (Bio-Rad) at 1:10000 for $10 \mathrm{~min}$, and samples were then rinsed three times. Bright light was avoided when working with the secondary antibody to minimize photo bleaching of the fluorescent dye. Finally, samples were mounted with fluorescent mounting medium, incubated until dry, and visualized under a immunofluorescence microscope (Olympus BX51, Japan). A negative control was set up, which did not include the primary antibody. Immunoreactive signals were visualized using diaminobenzidine (Sigma) as the substrate. Sections were counterstained with hematoxylin-eosin.

\section{RESULTS}

\section{Cloning and characterization of $C$. quadricarinatus Cyclin B cDNA}

Using degenerate PCR amplification, a cDNA fragment of 184 bp was obtained and, following a search for homologous sequences deposited in GenBank, was confirmed to be a partial sequence of the Cyclin B gene. The full-length cDNA of Cyclin B was successfully identified by RACE-PCR. Cyclin $B$ was $1779 \mathrm{bp}$ in length and contained an open reading frame (ORF) of $1203 \mathrm{bp}$, corresponding to 401 amino acid residues and 5' and 3' UTRs (untranslated region) of 72 and 504 bp, respectively (accession No. JX880246.1, Figure 1). The predicted molecular mass and pI value of the deduced Cyclin B protein were 45.1 and $8.84 \mathrm{kDa}$, respectively.

Two cyclin domains were identified as the deduced amino acid sequences of $C$. quadricarinatus Cyclin B. Furthermore, some sites or domains characteristic for Cyclin B were found to be highly conserved in C. quadricarinatus Cyclin B (Figure 1). These conserved domains included two cytoplasmic polyadenylation elements CPEs (U/AUUUUAU/A), one K-box (TGTGAT), one signature motif (position 170-204aa), the cyclin destruction box (RXALGXIXN) (position 34-42aa), which was necessary for Cyclin B to inhibit cell division from the middle to the late period, and a specific AMP-dependent PKA site (FLRRXSK) (position 275-281aa) (Minshull et al., 1989). 


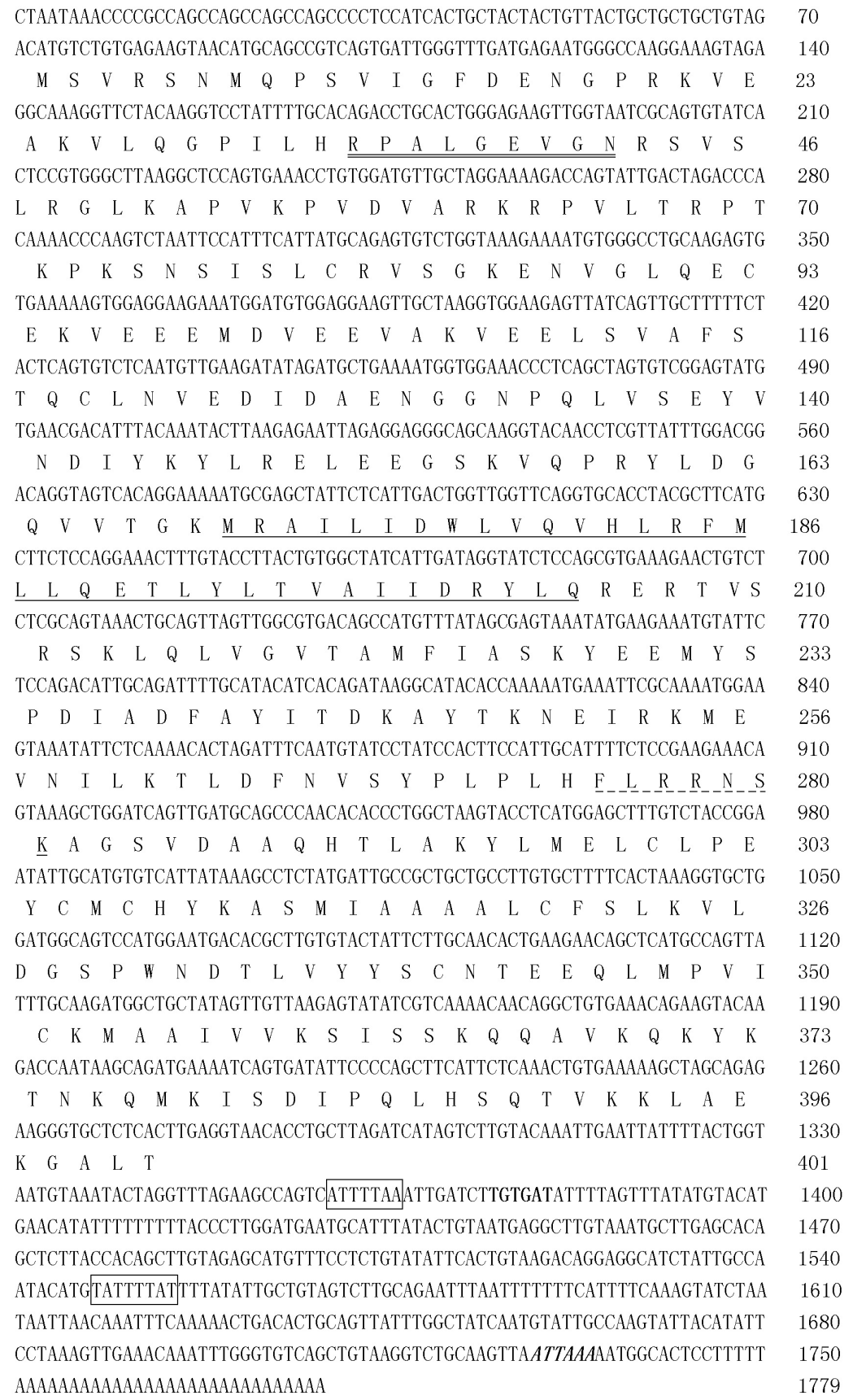

Figure 1. Nucleotide and deduced amino acid sequences of Cherax quadricarinatus Cyclin B. The eukaryotic potential polyadenylation signal ATTAAA is boldfaced and italicized. Two cytoplasmic polyadenylation elements, CPEs (U/AUUUUAU/A) and a K-box (TGTGAT) are marked by squares and boldfaced, respectively. The cyclin destruction box (RXALGXIXN), signature motif and PKA site (FLRRXSK) are s double-underlined, underlined, and broken underlined, respectively. 


\section{Multiple sequence alignment and phylogenetic analysis}

Homology between C. quadricarinatus Cyclin B and deduced amino acid sequences of Cyclin B from other species was explored via multiple sequence alignment using ClustalX. The C. quadricarinatus Cyclin B shared 69\% similarity with Metapenaeus ensis (GenBank accession No. ADI86225.1) and Metapenaeus affinis (ADI86226.1), 68\% with Marsupenaeus japonicus (AAV37462.1) and Penaeus monodon (ACH72068.1), 65 and 64\% with Macrobrachium rosenbergii (ADP95148.1) and Marsupenaeus japonicus (ADB44902.1), respectively, and 63 and 60\% with Scylla paramamosain (ACN54752.1) and E. sinensis (ACC77698.1), respectively. Homology with Dreissena polymorpha (AAC35952.1) and Haliotis diversicolor supertexta (ADP06655.1) was lower at 48 and 47\%, respectively, and that with Xenopus laevis (NP_001081268.1), Danio rerio (AAH45937.1), Gallus gallus (NP_001004369.1), and Mus musculus (NP_031656.2) Cyclin B2 was 47, 46, 45, and 43\%, respectively.

Phylogenetic analysis of Cyclin B from representative crustaceans, mollusks, mammals, and fishes (Figure 2) produced an NJ-phylogenetic tree that contained three distinct branches. Here, the C. quadricarinatus Cyclin B clustered with those of crustaceans and then mollusks, while sequences from mammals and fishes formed a separate cluster.

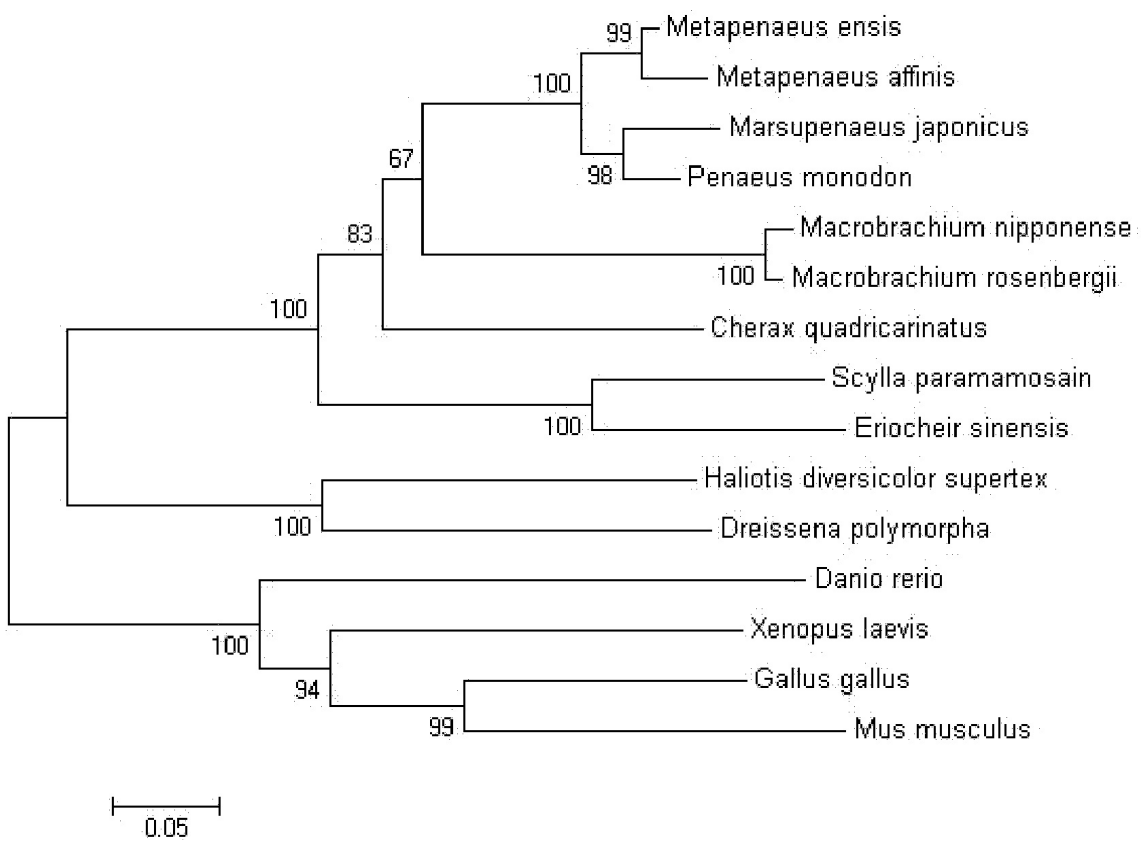

Figure 2. Neighbor-joining phylogenetic tree of the Cyclin B protein using the deduced amino acids sequences of Cherax quadricarinatus and other species obtained from GenBank database under the following accession No.: Metapenaeus ensis (ADI86225.1); Metapenaeus affinis (ADI86226.1); Marsupenaeus japonicus (AAV37462.1); Penaeus monodon (ACH72068.1); Macrobrachium rosenbergii (ADP95148.1); Marsupenaeus japonicus (ADB44902.1); Scylla paramamosain (ACN54752.1); Eriocheir sinensis (ACC77698.1); Dreissena polymorpha (AAC35952.1); Haliotis diversicolor supertexta (ADP06655.1); Xenopus laevis Cyclin B2 (NP_001081268.1); Danio rerio Cyclin B2 (AAH45937.1); Gallus gallus Cyclin B2 (NP 001004369.1); Mus musculus Cyclin B2 (NP_031656.2). The numbers in the phylogram nodes indicate percent bootstrap support for the phylogeny. The bar at the bottom indicates $2 \%$ amino acid divergence in sequences. 


\section{Pattern of Cyclin B expression}

As shown in Figure 3, the relative expression of Cyclin B mRNA in ovarian tissue was significantly higher than that in other tissues $(\mathrm{P}<0.05)$. Cyclin $\mathrm{B}$ was more abundantly expressed in ovaries than in embryos $(\mathrm{P}<0.01)$ (Figures 4 and 5). As the development of ovaries progressed, the expression of Cyclin $\mathrm{B}$ mRNA decreased $(\mathrm{P}<0.05)$ (Figure 4). Furthermore, the level of Cyclin B mRNA also decreased as embryonic development progressed $(\mathrm{P}<0.05)$. The highest expression of Cyclin B mRNA was found in stages I, with lower levels in stages II and III, and the lowest levels in stages IV and V (Figure 5).

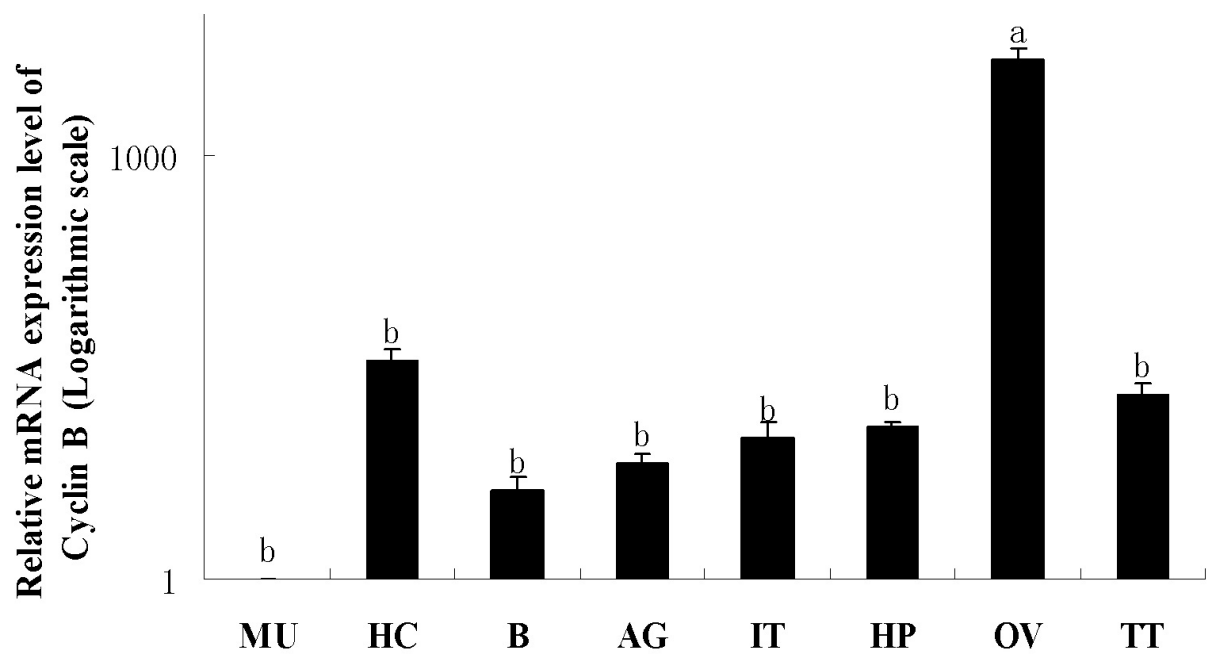

Figure 3. Tissue-dependent Cyclin B mRNA expression in Cherax quadricarinatus. ovaries (OV), testes (TT), antennal gland (AG), muscle (MU), hepatopancreas (HP), hemocytes (HC), intestine (IT), and brain (B).

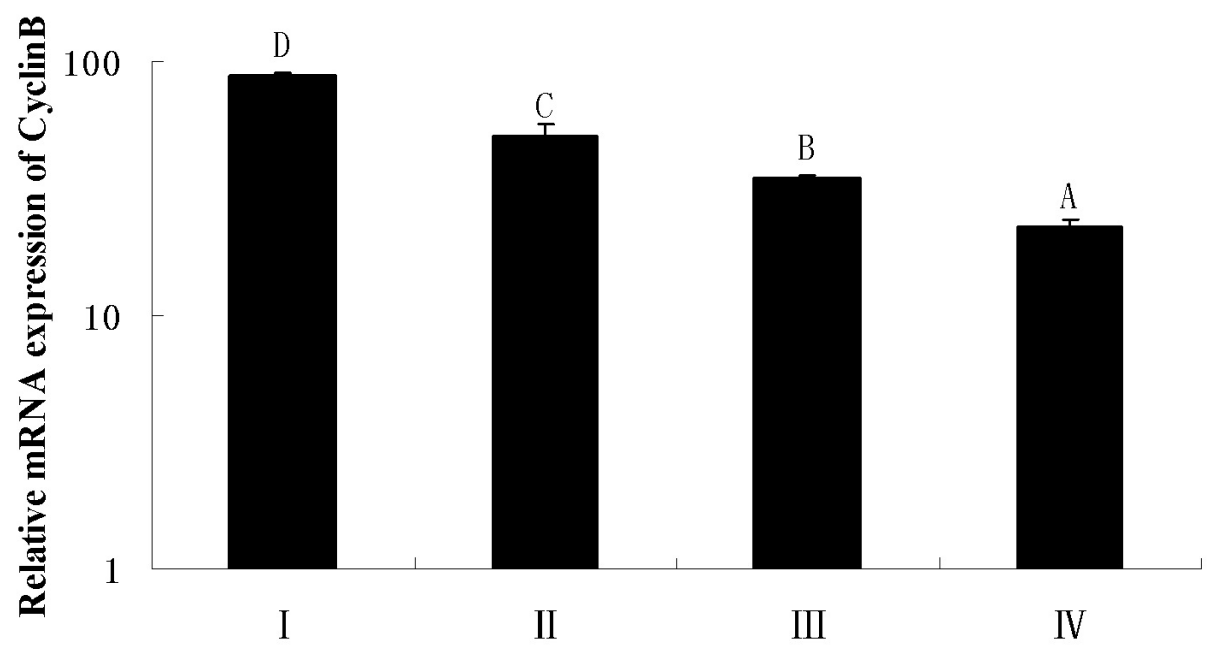

Figure 4. Relative expression of Cyclin B transcripts at different stages of ovarian development. 


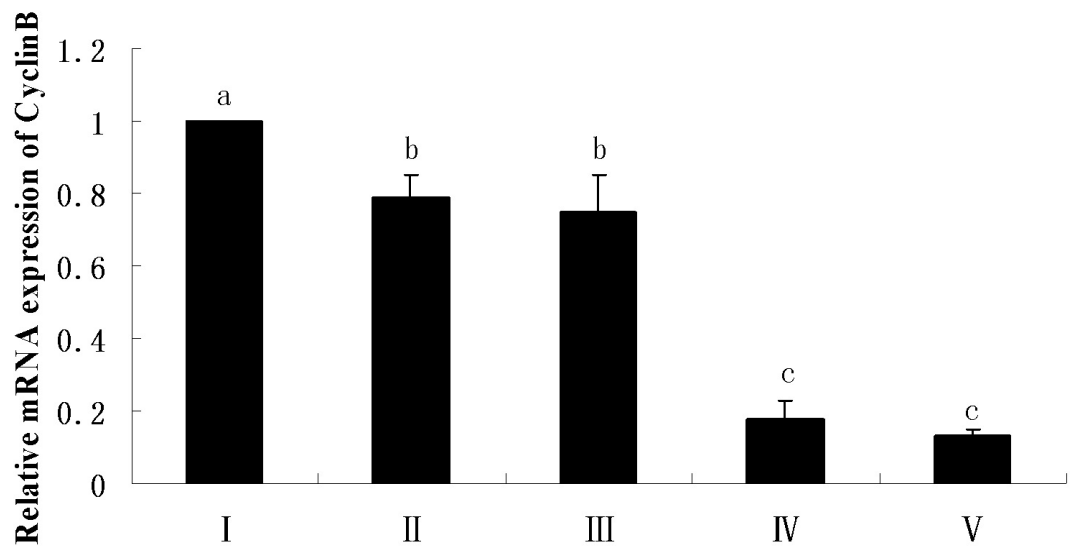

Figure 5. Relative expression of Cyclin B transcripts at different stages of embryonic development.

\section{Localization of Cyclin B in ovaries}

Localization of the Cyclin B protein was studied by IF. Specificity of the Cyclin B rabbit monoclonal antibody for C. quadricarinatus Cyclin B was confirmed in a preliminary test. Immunoreactive Cyclin B is shown in green. As the development of oocytes progressed, the signals became weaker in the ooplasm and stronger in the nucleus (Figure 6A and B). No positive signal was found in the negative control, which was not treated with the primary antibody.

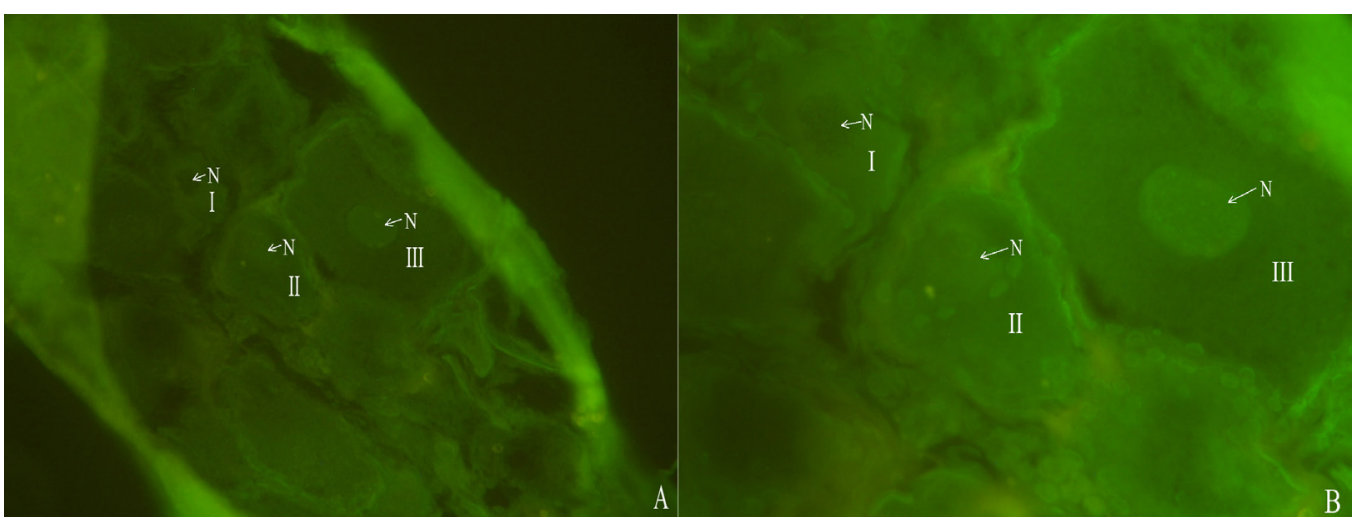

Figure 6. Immunofluorescence localization of Cyclin B protein in Cherax quadricarinatus ovary. Positive immunoreactive signals are shown in green. I, stage I oocyte; II, stage II oocyte; III stage III oocyte. N, nuclear. Magnification 200X for A; 400X for B.

\section{DISCUSSION}

In this study, the full-length cDNA of the C. quadricarinatus Cyclin B gene was characterized for the first time. Characteristic Cyclin B domains were found to be highly conserved (Figure 1). However, a polyadenylation signal sequence (AATAAA), which had previously been found in E. sinensis (Fang and Qiu, 2009), P. monodon (Qiu et al., 2007; Visudtiphole et al., 2009), M. japonicus (Qiu and Yamano, 2005), and M. nipponense (Wu, 2009) was ab- 
sent within 30 nucleotides of the poly(A)-tail in C. quadricarinatus. The sequence ATTAAA, which was found within 30 nucleotides of the poly(A)-tail in this study, might be a potential polyadenylation signal sequence (MacDonald and Redondo, 2002). The sequence AATATA, which is similar to the polyadenylation signal sequence (AATAAA), was also only found within 30 nucleotides of the poly(A)-tail in the M. rosenbergii Cyclin B gene (Fang, 2008).

To date, at least two cyclin B subtypes (Cyclin B1 and Cyclin B2) have been discovered in vertebrates. Only a single B-type cyclin with a 3'UTR of $504 \mathrm{bp}$ was identified in the present study. This is similar to that observed in M. nipponense (Wu, 2009), M. rosenbergii (Fang, 2008), and E. sinensis (Fang and Qiu, 2009), which also have long 3'UTRs consisting of 989, 1014, and $2400 \mathrm{bp}$, respectively. To our knowledge, the existence of Cyclin B2 has not been reported in invertebrates. In contrast, three forms of Cyclin B generated by 3'UTRs of three different lengths were reported in M. japonicus (Qiu and Yamano, 2005) and P. monodon (Visudtiphole et al., 2009). These studies indicated that the presence of various Cyclin B forms as a result of 3'UTR polymorphism might involve post-transcriptional regulation of gene expression and cellular and subcellular localization of the transcripts (Dalby and Glover, 1992; Qiu and Yamano, 2005; Visudtiphole et al., 2009).

qRT-PCR analysis showed that Cyclin B transcripts were ubiquitously distributed in various tissues of $M$. nipponense, and that high-levels were found in ovaries (Wu, 2009). In E. sinensis, Cyclin B transcripts were highly expressed in the testes and ovaries, and were expressed at lower levels in heart tissue, but were not detected in muscle, gill, and hepatopancreas tissue when examined by RT-PCR (Qiu and Liu, 2009). In M. rosenbergii, high levels of Cyclin B transcripts were also detected in the testes and ovaries and at lower levels were observed in the heart, hepatopancreas, gills, and muscle tissue (Fang, 2008). Moreover, $P$. monodon examined by RT-PCR revealed that cyclin B transcripts were most abundantly expressed in ovaries, followed by the testes, pleopods, and stomach, and low levels of expression were observed in the eyestalk, heart, and intestinal tissue (Visudtiphole et al., 2009). In the present study, Cyclin B transcripts were also found ubiquitously in all tissues, but the level of expression in ovarian tissue was significantly higher than that in other tissues when examined by qRT-PCR (Figure 3). The differences observed in the tissue distribution pattern might be due to qRT-PCR having higher sensitivity than RT-PCR, and differences between species (Wu, 2009). Differences in testicular expression in different species are probably due to the samples being obtained at different developmental stages (Wang et al., 2013b). However, the level of Cyclin B mRNA was consistently high in the ovaries, indicating that Cyclin B plays an important role in crustacean ovarian development as a component of MPF.

The detailed spatio-temporal expression profiles of C. quadricarinatus Cyclin B were further investigated during oogenesis and embryonic development. Cyclin B mRNA was more abundantly expressed in ovaries than in embryos $(\mathrm{P}<0.01)$. However, the expression patterns of Cyclin B transcripts during oogenesis differ between various crustacean species. The levels of Cyclin B expression during the reproductive cycle of M. japonicus as analyzed by qRT-PCR were not significantly different (Qiu and Yamano, 2005). In P. monodon, the level of Cyclin $\mathrm{B}$ expression in stage IV ovaries was significantly greater than that in stage I (primary vitellogenesis), but not in stages II (vitellogenesis), or III (cortical rod) (Visudtiphole et al., 2009). The best explanation for this is that the total RNA was isolated from ovarian tissue contains various developmental stages of oocytes, since $M$. japonicus and $P$. monodon spawn repeatedly during a single breeding season, and, therefore, even though mature, ovaries also contain immature oocytes as noted by Qiu and Yamano (2005). However, semi-quantitative RT-PCR 
analysis revealed that the amount of Cyclin B mRNA in M. rosenbergii was significantly higher during the previtellogenic and late vitellogenic stages, and was low during the early and middle vitellogenic stages (Fang, 2008). In M. nipponense, qRT-PCR revealed that the Cyclin B transcript is present in relatively high quantities at the perinucleolus stage, decreases at the nucleolus fusion stage, increases slightly at the oil globule stage, peaks during the yolk granule stage, and then drops remarkably at the paracmasis stage (Wu, 2009). In E. sinensis, the level of the cyclin B transcript is highest in stage I, drops remarkably at stage II, and reaches the lowest level at stage III, after which it begins to increase from stage IV until high expression is observed at the germinal vesicle breakdown (GVBD) stage (Fang and Qiu, 2008). The difference in Cyclin B expression might be associated with oogonial proliferation (mitosis) and oocyte meiotic maturation (Fang, 2008; Wu, 2009). In the present study, the color of the ovaries at each development stage was the same, and the pattern of Cyclin B mRNA expression in the ovaries decreased during oogenesis. At stages I and II, the shrimp oogonia undergo active mitosis and their number increases, and the level of Cyclin B mRNA significantly increased. At stages III and IV, the growing oocytes accumulate yolk and there are low levels of proliferation and the cell cycle is arrested at the first meiotic prophase, and during this phase, the level of Cyclin B mRNA falls to the lowest level. Following yolk accumulation, the fully-grown oocytes resume meiosis and GVBD occurs at the final maturation stage. The level of Cyclin B mRNA may increase again, which we could not detect because the ovary stage IV preceded the final whole maturation stage.

In the present study, immunofluorescence analysis revealed that the Cyclin B protein relocated from the cytoplasm to the nucleus during ovarian development (Figure 6A and B). Similar results have been reported for Cdc2 in E. sinensis (Qiu and Liu, 2009) and C. quadricarinatus (Wang et al., 2013b). In M. japonicus, in situ hybridization showed that the short transcript was expressed in the ova as early as the oogonia stage and that most accumulation occurred at the perinucleolus stage (Qiu and Yamano, 2005). Previous studies in mice and starfish demonstrated that intracellular relocation of Cyclin B-associated Cdc2 kinase is an essential step for acquiring meiotic resumption competence (Ookata et al., 1992) and induction of GVBD (Mitra and Schultz, 1996). The relocated Cyclin B protein in C. quadricarinatus is most likely an activated form associated with Cdc2 kinase, and the activated MPF mediates the dramatic changes that are required in nuclear and cytoskeletal architecture for accurate chromosome segregation as reported by Qiu and Liu (2009) and Wang et al. (2013b) in E. sinensis and C. quadricarinatus.

In conclusion, we successfully cloned and sequenced the full-length cDNA of Cyclin B, characterized the mRNA distribution in various tissues, and investigated the pattern of expression in different stages of gonadal and embryonic development in Cherax quadricarinatus. Cyclin B mRNA was mainly expressed in the ovaries and its levels decreased during ovarian development. Immunofluorescence analysis revealed that the Cyclin B protein relocated from the cytoplasm to the nucleus over the course of ovarian development.

\section{Conflicts of interest}

The authors declare no conflict of interest.

\section{ACKNOWLEDGMENTS}

Research supported by grants from the National Natural Science Foundation of China 
(\#31172043) and the Shanghai Municipal Science and Technology Commission Research Project (\#12391900700).

\section{REFERENCES}

Abdu U, Yehezkel G and Sagi A (2000). Oocyte development and polypeptide dynamics during ovarian maturation in the red-claw crayfish Cherax quadricarinatus. Invertebr. Reprod. Dev. 37: 75-83.

Abdu U, Davis C, Khalaila I and Sagi A (2002). The vitellogenin cDNA of Cherax quadricarinatus encodes a lipovitellin with calcium binding ability, and its expression is induced following the removal of the androgenic gland in a sexually plastic system. Gen. Comp. Endocr. 127: 263-272.

Dalby B and Glover DM (1992). 3'non-translated sequences in Drosophila cyclin B transcripts direct posterior pole accumulation late in oogenesis and peri-nuclear association in syncytial embryos. Development 115: 989-997.

Fang JJ (2008). Molecular cloning of Cyclin B transcript and its expression analysis during oogenesis in the Chinese mitten crab (Eriocheir sinensis) and Malaysian prawn (Macrobrachium rosenbergii). Master's thesis, Shanghai Ocean University, Shanghai.

Fang JJ and Qiu GF (2009). Molecular cloning of cyclin B transcript with an unusually long 3' untranslation region and its expression analysis during oogenesis in the Chinese mitten crab, Eriocheir sinensis. Mol. Biol. Rep. 36: 1521-1529.

Lees EM and Harlow E (1993). Sequences within the conserved cyclin box of human cyclin A are sufficient for binding to and activation of cde2 kinase. Mol. Cell. Biol. 13: 1194-1201.

Li CJ, Vassilev A and DePamphilis ML (2004). Role for Cdk1 (Cdc2)/cyclin A in preventing the mammalian origin recognition complex's largest subunit (Orc1) from binding to chromatin during mitosis. Mol. Cell Biol. 24: 5875-5886.

Li JY, Zhao YL, Qin F and Xu XQ (2007). Study on the ultrastructural of vitellogenesis of Cherax quadricarinatus. J. Fudan Univ. 46: 987-991.

Li JY, Guo ZL, Gan XH, Wang Q, et al. (2010). Biochemical changes during vitellogenesis in the red claw crayfish, Cherax quadricarinatus (von Martens). Aquac. Res. 41: e446-e455.

Li JY, Guo ZL, Gan XH, Wang L, et al. (2011). Effect of different dietary lipid sources on growth and gonad maturation of pre-adult female Cherax quadricarinatus (von Martens). Aquacult. Nutr. 17: e853-e860.

Livak KJ and Schmittgen TD (2001). Analysis of temporal gene expression data using real-time quantitative PCR and the 2[-Delta Delta C (T)] Method. Methods 25: 402-408.

MacDonald CC and Redondo JL (2002). Reexamining the polyadenylation signal: were we wrong about AAUAAA? Mol. Cell. Endocrinol. 190: 1-8.

Minshull J, Blow JJ and Hunt T (1989). Translation of cyclin mRNA is necessary for extracts of activated Xenopus eggs to enter mitosis. Cell. 56: 947-956.

Minshull J, Golsteyn R, Hill CS and Hunt T (1990). The A- and B-type cyclin associated cdc2 kinases in Xenopus turn on and off at different times in the cell cycle. EMBO J. 9: 2865-2875.

Mitra J and Schultz RM (1996). Regulation of the acquisition of meiotic competence in the mouse: changes in the subcellular localization of cdc2, cyclin B1, cdc25C and wee1, and in the concentration of these proteins and their transcripts. J. Cell Sci. 109: 2407-2415.

Ookata K, Hisanaga S, Okano T, Tachibana K, et al. (1992). Relocation and distinct subcellular localization of p34cdc2cyclin B complex at meiosis reinitiation in starfish oocytes. EMBO J. 11: 1763-1772.

Qiu GF and Yamano K (2005). Three forms of cyclin B transcripts in the ovary of the kuruma prawn Marsupenaeus japonicus: their molecular characterizations and expression profiles during oogenesis. Comp. Biochem. Physiol. B. 141: 186-195.

Qiu GF and Liu P (2009). On the role of Cdc2 kinase during meiotic maturation of oocyte in the Chinese mitten crab, Eriocheir sinensis. Comp. Biochem. Physiol. B. 152: 243-248.

Qiu L, Jiang S, Zhou F, Huang J, et al. (2007). Molecular cloning and. characterization of a cyclin B gene on the ovarian maturation stage of black tiger shrimp (Penaeus monodon). Mol. Biol. Rep. DOI 10.1007/s11033-006-9052-4.

Rodríguez-González H, Hernández-Llamas A, Villarreal H, Saucedo PE, et al. (2006). Gonadal development and biochemical composition of female crayfish Cherax quadricarinatus (Decapoda: Parastacidae) in relation to the Gonadosomatic Index at first maturation. Aquaculture 254: 637-645.

Rodríguez-González H, Villarreal H, García-Ulloa M and Hernández-Llamas A (2009a). Evaluation of practical diets containing different protein levels on gonad development of female redclaw crayfish, Cherax quadricarinatus. Aquacult. Nutr. 15: 347-355.

Rodríguez-González H, Villarreal H, García-Ulloa M and Hernández-Llamas A (2009b). Dietary lipid requirements for 
optimal egg quality of redclaw crayfish. Cherax quadricarinatus. J. World Aquacult. Sot. 40: 531-539.

Sagi A, Shoukrun R, Khalaila I and Rise M (1996). Gonad maturation, morphological and physiological changes during the first reproductive cycle of the crayfish Cherax quadricarinatus female. Invertebr. Reprod. Dev. 29: 235-242.

Vazquez FJ, Tropea C and López Greco LS (2008). Differentiation of the female reproductive system and the onset of maturity in the freshwater crayfish "red claw" Cherax quadricarinatus (von Martens, 1898) (Decapoda, Parastacidae) through a macroscopic and microscopic approach. Inv. Biol. 127: 433-443.

Visudtiphole V, Klinbunga S and Kirtikara K (2009). Molecular characterization and expression profiles of cyclin A and cyclin B during ovarian development of the giant tiger shrimp Penaeus monodon. Comp. Biochem. Physiol. A. 152: 535-543.

Wang LM, Zuo D, Lv WW, Li JY, et al. (2013a). Effects of dietary soybean lecithin on gonadal development and vitellogenin mRNA expression in the female redclaw crayfish, Cherax quadricarinatus (von Martens) at first maturation. Aquacult. Res. 44: 1167-1176.

Wang LM, Zuo D, Lv WW, Wang DL, et al. (2013b). Characterization of Cdc2 kinase in the red claw crayfish (Cherax quadricarinatus): Evidence for its role in regulating oogenesis. Gene. 515: 258-265.

Wu P (2009). Analysis of ovary ESTs and cDNA Cloning and Expression Profile of Reproduction-related Genes from Macrobrachium nipponense. Doctoral thesis East China Normal University, Shangai. 